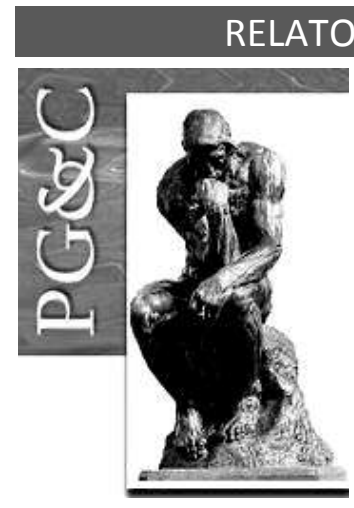

\title{
SMART CAMPUS NO BRASIL: A PERCEPÇÃO DOS GESTORES DAS IFES
}

\author{
Lucilene Klenia Rodrigues Bandeira \\ Doutora em Administração pela Université Grenoble Alpes, França. \\ Professora da Universidade Federal da Paraíba, Brasil. \\ E-mail: klenia.bandeira@gmail.com \\ Mário de Sousa Araújo Neto \\ Mestre em Engenharia Elétrica pela Universidade do Porto, Portugal. \\ Engenheiro Eletricista da Universidade Federal de Campina Grande, Brasil. \\ E-mail: msaneto@gmail.com
}

\begin{abstract}
Resumo
O objetivo desta pesquisa é de investigar a percepção dos gestores das instituições federais de ensino superior do Brasil sobre o conceito e a criação de um smart campus. Os últimos vinte anos apresentaram uma evolução na área das tecnologias da informação e da comunicação, que vem promovendo importantes mudanças na forma de trabalho das pessoas, no transporte, na comunicação, na educação, no setor industrial etc. O smart campus é um conceito emergente, que se originou no conceito de smart cities, o qual aplica nas cidades as referidas tecnologias, com o objetivo de enfrentar os desafios e as oportunidades cotidianos. Nesse contexto, o campus é visto como uma cidade e enfrenta problemas similares aos de uma cidade convencional. Trata-se de uma pesquisa exploratória de abordagem quantitativa. A pesquisa foi realizada com gestores de 27 instituições, durante o Fórum Nacional de Pró-Reitores de Planejamento e de Administração das Instituições Federais de Ensino Superior (FORPLAD). Foram analisadas seis dimensões sobre o smart campus: importância; conhecimento dos gestores sobre smart campus; estrutura da instituição; gestão; equipe e resultados esperados do smart campus. Trata-se de um tema de pesquisa recente, e isso se reflete nos resultados, pois a maioria dos gestores desconhecem o conceito, porém tem uma percepção geral muito positiva sobre a criação do smart campus em suas instituições. Os estudos sobre smart campus se concentram nas áreas de tecnologia, todavia podem ser uma oportunidade de pesquisa para a área da Administração, focando, sobretudo, estudos a respeito da governança do smart campus.
\end{abstract}

Palavras-chave: Smart campus. Gestores. Fórum Nacional de Pró-Reitores de Planejamento e de Administração das Instituições Federais de Ensino Superior - FORPLAD. Percepção.

\section{SMART CAMPUS IN BRAZIL: THE PERCEPTION OF IFES MANAGERS}

\begin{abstract}
The aim of the research is to identify the perception of managers of federal higher education institutions in Brazil about the concept and the creation of a smart campus. The last twenty years have shown an evolution in the area of information and communication technologies and have been promoting important changes in the way people work, transportation, communication, education, industrial sector, etc. The smart campus is an emerging concept that has its origin in the concept of smart cities, which applies these technologies in cities in order to better meet daily challenges and opportunities. In this context, the campus is seen as a city and faces similar problems to a conventional city. It is an exploratory research of the quantitative type. The survey was conducted with managers from 27 institutions during the National Forum of Pro-Rectors of Planning and Administration of Federal Higher Education Institutions (FORPLAD). Six dimensions on the smart campus were analyzed: Importance; Knowledge of managers about Smart Campus; Institution structure; Management; Team; Smart Campus
\end{abstract}

Perspectivas em Gestão \& Conhecimento, João Pessoa, v. 10, n. 3, p. 189-204, set./dez. 2020. DOI: https://dx.doi.org/10.22478/ufpb.2236-417X.2020v10n3.48609

http://periodicos.ufpb.br/ojs2/index.php/pgc. ISSN: 2236-417X. Publicação sob Licença (cc) EY-Nc-ND 
expected results. This is a recent research topic and this is reflected in the results, most managers are unaware of the concept, but have a very positive overall perception about the creation of smart campus in their institutions. Smart campus studies focus on technology, but it can be a research opportunity for administration, focusing primarily on smart campus governance studies.

Keywords: Smart campus. Managers. Forum of Pro-Rectors of Planning and Administration of Federal Higher Education Institutions - FORPLAD. Perception.

\section{INTRODUÇÃO}

Nos últimos anos, o crescimento explosivo do conhecimento levou a uma variedade de maneiras de difundir o conhecimento e a educação. A transformação do modelo de educação induziu o smart campus, que implementa a educação combinando informações e tecnologia da informação para atender às diversas necessidades de estudantes e universidades (XIA et al., 2018).

A relação do conceito de smart city aplicado ao campus tem sido feita com foco nas tecnologias que podem ser utilizadas nas universidades, mas esse modelo também pode se aplicar em outras Instituições de ensino e/ou de pesquisa. No caso das Instituições de Ensino Superior, o campus é considerado uma cidade, entende-se que elas vivem a maior parte dos desafios das cidades convencionais, daí o interesse em aplicar soluções inteligentes para elas. Como criar e gerenciar um smart campus? Partindo do princípio de que, nas universidades, muitos departamentos estão desenvolvendo tecnologias de ponta em áreas que fazem parte do modelo smart campus, como trazer essas tecnologias desenvolvidas internamente para o consumo do próprio campus? Em outros termos, qual é a estrutura de governança adequada para o campus inteligente?

Como tudo isso ainda é muito novo, não encontramos estudos que abordem claramente a relação entre a gestão universitária e o smart campus, os desafios para os gestores criarem um smart campus ou ainda sobre a percepção dos gestores sobre smart campus. Identificamos ações voltadas para aplicação das tecnologias de compartilhamento de informação como aplicativos de mobilidade, (TORRES-SOSPEDRA et al., 2015), o smart campus da Facens (ROMANO et al., 2016), a plataforma educacional virtual (CHEN \& ZHANG, 2012), o monitoramento de consumo de energia elétrica, (DE ANGELIS et al., 2015), um aplicativo de compartilhamento de bicicletas, (DE SÁ et al., 2016), a criação de uma rede inteligente de sensores para estacionamento de veículos, (BANDARA et al., 2016), entre outros.

As propostas em geral são mais superficiais no tocante as dimensões gerenciais do campus, elas se concentram nas tecnologias desenvolvidas e nos resultados esperados. Portanto, ao identificar esse gap teórico, o objetivo da pesquisa foi o de investigar a percepção dos gestores das Instituições Federais de Ensino Superior-IFES sobre o conceito e a criação de um smart campus. As seguintes dimensões foram consideradas para a pesquisa: importância do modelo smart campus; conhecimento dos gestores sobre smart campus; estrutura da instituição; gestão; equipe e resultados esperados do smart campus.

Sabe-se que existem muitos novos aplicativos e serviços de aprendizado nos campi inteligentes (XIA et al., 2018). Entretanto, o smart campus é um desafio gerencial para as universidades brasileiras. Quanto à estrutura, este texto traz, na sequência, o conceito de smart campus, uma abordagem sobre alguns desafios na gestão das universidades brasileiras, a metodologia empregada, além dos resultados da pesquisa, a discussão e a conclusão.

Perspectivas em Gestão \& Conhecimento, João Pessoa, v. 10, n. 3, p. 189-204, set./dez. 2020. 


\section{SMART CAMPUS: UM CONCEITO RECENTE}

Recentemente, o smart campus se tornou um tópico de pesquisa importante, porque a rede do campus é uma instalação básica. Ele deve ser estruturado por redes rápidas, fortes e seguras no campus (WANG et al., 2018) e é uma nova etapa na criação de tecnologias de informação em faculdades e universidades. Suas características incluem onipresença de alta velocidade da Internet, Internet das Coisas, interconexão contínua e consciência ambiental, ampla popularização e aplicação de terminais inteligentes e aprendizagem colaborativa aberta no ambiente de pesquisa e de trabalho. 0 campus inteligente está intimamente integrado às novas tecnologias da Internet, que usam computação em nuvem, virtualização, big data e a Internet das Coisas para mudar as formas de interagir, transferir conhecimentos e de compartilhar recursos entre os usuários do campus (NAN et al., 2018).

O conceito de smart campus é baseado no conceito de smart city. O campus universitário é uma pequena cidade que tem os mesmos problemas e desafios de uma cidade convencional. Technopolis ou smart city é um conceito que foi desenvolvido nos anos de 1990, referente a tecnologias globais voltadas para as cidades conectadas em uma sofisticada rede de comunicação para deixar a economia mundial cada vez mais integrada (GIBSON; KOZMETSKY; SMILOR, 1992). Uma cidade inteligente usa tecnologia de informação e comunicação (TIC) para melhorar sua habitabilidade, a capacidade de trabalho e a sustentabilidade. Primeiro, uma cidade inteligente coleta informações sobre si mesma por meio de sensores, de outros dispositivos e dos sistemas existentes. Em seguida, comunica esses dados usando redes com ou sem fio e, terceiro, analisa-os para entender o que está acontecendo em certo momento e o que provavelmente acontecerá em seguida (SMART CITY COUNCIL). As diferentes abordagens do conceito de smart city dificulta que se chegue a uma definição única e inequívoca de cidade inteligente (TORRES-SOSPEDRA, 2015). O smart campus revela-se como uma estrutura nova baseada em uma combinação de ferramentas de alta tecnologia para realizar, de maneira inteligente, segura e sustentável, as atividades cotidianas e as novas formas de trabalho para o ensino e a gestão. Entende-se que as tecnologias de informação e de comunicação estão no "coração" dos dois modelos. Dessa forma, esta pesquisa dá ênfase a esse aspecto para entender a relação entre os conceitos de smart city e de smart campus. Pela própria natureza de suas origens tecnológicas, trata-se de um conceito emergente na literatura (ZHIHONG et al., 2018). O próprio termo Internet das Coisas foi usado pela primeira vez por Kevin Ashton, em 1999. O Quadro 1 apresenta alguns conceitos de smart campus.

Quadro 1 - Conceitos de smart campus

\begin{tabular}{|l|l|}
\hline \multicolumn{1}{|c|}{ Referência } & \multicolumn{1}{c|}{ Conceito } \\
\hline Ying et al. (2012) & $\begin{array}{l}\text { O campus digital é baseado em rede e utiliza meios e ferramentas } \\
\text { de informação avançados. Smart campus é um estágio avançado } \\
\text { na construção da informatização do campus, que pode tornar a } \\
\text { vida cotidiana no campus mais conveniente. }\end{array}$ \\
\hline Kwok (2015) & $\begin{array}{l}\text { Os elementos essenciais para a construção de campus inteligente } \\
\text { são: consideraçães de dados, conhecimento processual e } \\
\text { integração de sistemas. }\end{array}$ \\
\hline Xin et al. (2016) & $\begin{array}{l}\text { Apresentam três características necessárias a um smart campus: a } \\
\text { percepção precisa do contexto e o acesso onipresente à rede; a } \\
\text { alocação eficiente de recursos e a tomada de decisão inteligente } \\
\text { baseada em princípios objetivos. }\end{array}$ \\
\hline Ai-Min et al. (2018) & $\begin{array}{l}\text { Smart campus é um campus intelectualizado, aprendendo e } \\
\text { vivendo em um ambiente integrado e baseado na internet das } \\
\text { coisas. É uma plataforma integrada de serviços de informação, }\end{array}$ \\
\hline
\end{tabular}

Perspectivas em Gestão \& Conhecimento, João Pessoa, v. 10, n. 3, p. 189-204, set./dez. 2020. 


\begin{tabular}{|c|c|}
\hline & aberta, inovadora, colaborativa e inteligente. \\
\hline Xin et al. (2018) & $\begin{array}{l}\text { As características do campus inteligente incluem conscientização } \\
\text { ambiental abrangente, conexão de rede contínua, suporte maciço } \\
\text { a dados, ambiente de aprendizado aberto e serviços } \\
\text { personalizados para professores e alunos. }\end{array}$ \\
\hline Zhihua et al. (2018) & $\begin{array}{l}\text { A transformação do modelo de educação induziu o smart campus, } \\
\text { que implementa a educação através da combinação de informação } \\
\text { e tecnologia da informação, para atender às diversas necessidades } \\
\text { dos estudantes e das universidades. }\end{array}$ \\
\hline Sánchez Torres et al. (2018) & $\begin{array}{l}\text { Um smart campus é uma entidade de qualquer tipo que utiliza a } \\
\text { tecnologia para apoiar sua infraestrutura e seus processos, com o } \\
\text { fim de melhorá-los para as pessoas usarem. Ele não se limita a } \\
\text { melhorar a infraestrutura das universidades por meio da } \\
\text { tecnologia, pois seu fim principal é de melhorar a qualidade da } \\
\text { educação ministrada pelas instituições. }\end{array}$ \\
\hline Luo Li (2018) & $\begin{array}{l}\text { A essência de um campus inteligente é uma plataforma virtual } \\
\text { para a troca de informações. A tecnologia da Internet das coisas é, } \\
\text { frequentemente, necessária para ser usada ao máximo e construir } \\
\text { um sistema de rede gigante que pode fornecer pesquisa de } \\
\text { informações, troca de recursos e outros serviços para professores } \\
\text { e alunos e, finalmente, fazer o trabalho de gestão do campus } \\
\text { inteligente e em tempo real. }\end{array}$ \\
\hline Yi e Zixi (2018) & $\begin{array}{l}\text { O smart campus é uma forma mais inteligente e integrada, } \\
\text { baseada na fundação do 'campus digital'. Refere-se ao trabalho } \\
\text { baseado na Internet, aprendendo e vivendo o ambiente de } \\
\text { integração. Esse ambiente integrado é baseado em vários sistemas } \\
\text { de serviços de aplicativos e integra totalmente a medicina, a } \\
\text { gestão e a vida do campus. }\end{array}$ \\
\hline Jiwei (2018) & $\begin{array}{l}\text { O smart campus é proposto com o desenvolvimento da tecnologia } \\
\text { da Internet e, normalmente, usado para compreender, perceber, } \\
\text { adquirir e transmitir dados em massa. }\end{array}$ \\
\hline Zhihong et al. (2018) & $\begin{array}{l}\text { Smart campus é uma área de pesquisa interessante, nova e } \\
\text { emergente, que usa tecnologia e infraestrutura para apoiar e } \\
\text { melhorar seus processos nos serviços do campus, ensino, } \\
\text { aprendizagem e pesquisa. }\end{array}$ \\
\hline Yu et al. (2019) & $\begin{array}{l}\text { O smart campus está atraindo a atenção das pessoas porque se } \\
\text { adapta às necessidades de vários alunos. Isso é atribuído a } \\
\text { dispositivos com recursos de rede. A interoperabilidade é uma das } \\
\text { características mais importantes de um sistema de smart campus, } \\
\text { um sistema que tem a capacidade de conectar diversos } \\
\text { dispositivos eletrônicos para que eles funcionem como um sistema } \\
\text { unificado. }\end{array}$ \\
\hline Prandi et al. (2019) & $\begin{array}{l}\text { O conceito de smart campus é um refinamento do termo } \\
\text { 'ambiente inteligente', definido como um ambiente físico em que } \\
\text { tecnologias inovadoras e difundidas de informação e comunicação } \\
\text { possibilitam que as pessoas vivenciem e interajam com o espaço e } \\
\text { gerem dados. }\end{array}$ \\
\hline
\end{tabular}

Fonte: Elaborado pelos autores (2019)

Os conceitos se completam e focam no uso inteligente da tecnologia aplicada no campus para atender as suas necessidades. Assim, a essência de um campus inteligente é uma plataforma virtual para a troca de informações. A tecnologia da Internet das Coisas é necessária para ser usada ao máximo e construir um sistema de rede gigante, que pode

Perspectivas em Gestão \& Conhecimento, João Pessoa, v. 10, n. 3, p. 189-204, set./dez. 2020. 
proporcionar pesquisas de informações, trocas de recursos e outros serviços para professores e alunos e, finalmente, fazer o trabalho de gestão do campus em tempo real (LUO, 2018).

A estrutura do smart campus é baseada no uso da tecnologia da informação para promover um ambiente de aprendizagem mais eficiente, utilizando serviços digitais e sociais (ATIF et al., 2015; BARBATO et al., 2016; XIN et al., 2016; BATES; FRIDAY, 2017). No Brasil, citase a FACENS, uma instituição privada que, desde 2014, desenvolveu e implantou o modelo de smart campus. O projeto foi submetido a um programa internacional, o Global Entrepreneurship Lab, promovido pelo MIT Sloan-School of Management, que foi selecionado e recebeu a assessoria necessária para ser implantado. As áreas de atuação desse projeto são as seguintes: educação, energia, indústria e negócios, meio ambiente, mobilidade e segurança, saúde e qualidade de vida, tecnologia da informação e comunicação, urbanização e o núcleo facilitador. Neste caso, o foco do projeto é no ensino, na inovação e no empreendedorismo. Apresenta-se uma abordagem do tipo Learning Management Systems, com o desenvolvimento de protótipos para atender às necessidades do mercado (ROMANO; SIRION; PACHECO, 2016). É importante enfatizar que, nos casos em que existe a semelhança de perfil, de desafios e de oportunidades de desenvolvimento, iniciativas como esta pode ser um catalisador de soluções compartilhadas ou de desenvolvimento de trabalho colaborativo inter ou intrainstituições. As universidades se encaixam nesse contexto, pois têm estruturas organizacionais, desafios e propósitos similares. Entretanto, a forma como gerenciam é que influencia os resultados esperados. Finalmente, o smart campus se caracteriza como uma estrutura nova baseada em uma combinação de ferramentas de alta tecnologia para realizar, de maneira inteligente, segura e sustentável, as atividades cotidianas e as novas formas de trabalho para o ensino e a gestão. Por isso é importante compreender o conceito e sua aplicabilidade em diferentes contextos.

\section{GESTÃO DAS INSTITUIÇÕES FEDERAIS DE ENSINO SUPERIOR (IFES)}

O aumento da tecnologia também tem avançado para o setor educacional, fazendo com que o nível de informatização da universidade também melhore constantemente. No entanto, ainda existem muitos problemas na gestão tradicional do campus, razão por que a construção do smart campus é imperativa (YANG et al., 2018). A universidade está vendo a necessidade de promover práticas sociais, culturais, ambientais e econômicas, que sejam adequadas e orientadas para dar conta dos desafios de seu tempo. Para isso, tem procurado se organizar para ir além de suas tarefas básicas de produção de conhecimentos e de formação profissional e considera também os impactos que suas atividades podem gerar em si mesma, em sua comunidade interna e em seus stakeholders (RIBEIRO, 2014).

A gestão das universidades é um desafio para os gestores. A rígida legislação para a execução orçamentária, as diversas interpretações dos órgãos de controle, a expansão universitária e o despreparo para enfrentar as atuais restrições orçamentárias são apenas algumas das dificuldades que eles enfrentam. Um gestor do setor privado, por exemplo, tem condições de barganhar, no mundo globalizado e conectado, seu melhor fornecedor, a melhor relação custo-benefício etc. O gestor público, muitas vezes, não consegue adquirir determinado produto ou serviço simplesmente porque seu campus de lotação fica em uma região pouco atrativa para os negócios de determinada indústria. Isso resulta em ofertas de produtos e serviços restritos a um grupo de fornecedores e, nem sempre, a ampla concorrência ou os princípios legais da administração pública são alcançados. Nesse contexto, é importante desenvolver uma gestão que crie ações internas que gerem tecnologias e recursos desenvolvidos e aplicados internamente para suprir as carências da própria instituição. Trata-se, nesse ponto da gestão da instituição, ou seja, das decisões ligadas ao seu funcionamento, de um negócio, e não, de um processo decisório complexo, com a participação

Perspectivas em Gestão \& Conhecimento, João Pessoa, v. 10, n. 3, p. 189-204, set./dez. 2020. 
de colegiados representativos compostos de diversas categorias da universidade. Por isso, entende-se que a governança corporativa pode ser uma forma de melhorar a gestão atual e promover ações inovadoras e eficientes, como, por exemplo, o smart campus.

A governança corporativa vem se destacando no cenário empresarial das últimas décadas e conquistando espaço nas Instituições de Ensino Superior que, além de enfrentar desafios no âmbito educacional, precisam adequar a gestão para aperfeiçoar seus processos e estratégias (MELLO; SILVA, 2018). A governança corporativa é o sistema pelo qual as empresas e demais organizações são dirigidas, monitoradas e incentivadas e que envolve os relacionamentos entre os sócios, o Conselho de Administração, a Diretoria, os órgãos de fiscalização e de controle e as demais partes interessadas. As boas práticas de governança corporativa convertem princípios básicos em recomendações objetivas e alinham interesses com a finalidade de preservar e otimizar o valor econômico de longo prazo da organização, facilitando seu acesso a recursos e contribuindo para melhorar a qualidade da gestão da organização, sua longevidade e o bem comum, com base nos seguintes princípios: transparência, equidade, prestação de contas (accountability) e responsabilidade corporativa (INSTITUTO BRASILEIRO DE GOVERNANÇA CORPORATIVA, 2018). O desafio consiste em saber qual é o modelo de governança adaptado para as universidades.

A atual infraestrutura das Instituições de Ensino Superior no Brasil tem condições de suportar um modelo de smart campus umas em maior nível outras menor nível de recursos disponíveis. A maioria das tecnologias, citadas nos conceitos acima, estão sendo pesquisadas ou desenvolvidas nessas Instituições. Um grande desafio gerencial seria agrupar o que está sendo feito internamente e transformar em benefício para o campus, como política Institucional. Uma espécie de "guarda-chuva" gerencial para promover e favorecer o desenvolvimento e uso de tecnologias para o campus, impedindo com isso que, cada pesquisador desenvolva isoladamente suas tecnologias para serem testadas ou utilizadas fora da Universidade.

\section{METODOLOGIA}

Para Silva e Menezes (2005, p. 20), "pesquisa é um conjunto de ações, propostas para encontrar a solução para um problema, que têm por base procedimentos racionais e sistemáticos". A pesquisa é realizada quando se tem um problema e não se têm informações para solucioná-lo. A escolha da metodologia de uma pesquisa deve ser coerente com seu objetivo. No tocante aos aspectos metodológicos desta pesquisa, podemos caracterizá-la como um estudo exploratório, por meio da qual o autor pode explorar um assunto que ainda é desconhecido ou pouco explorado (GIL, 2008). Trata-se de uma pesquisa de abordagem quantitativa de natureza aplicada. Os procedimentos adotados foram a pesquisa bibliográfica e de campo. Segundo Boccato (2006, p. 266), "a pesquisa bibliográfica busca a resolução de um problema (hipótese) por meio de referenciais teóricos publicados, analisando e discutindo as várias contribuições científicas". A pesquisa de campo caracteriza-se pelas investigações em que além da pesquisa bibliográfica e/ou documental, se coletam dados junto a pessoas (FONSECA, 2002).

A coleta de dados foi realizada durante o Fórum Nacional de Pró-Reitores de Planejamento e de Administração das Instituições Federais de Ensino Superior (FORPLAD), ocorrido no período de 17 a 19 de junho de 2019 na cidade de Campina Grande-PB. O públicoalvo eram os participantes do FORPLAD. Esse fórum é composto de pró-reitores de administração e de planejamento e seus respectivos adjuntos ou coordenadores de área. Esses servidores podem ser docentes ou técnico-administrativos que exercem cargos de direção nas instituições públicas federais. A amostra por instituição foi de 27 instituições em um universo de 63 (PORTAL DA TRANSPARÊNCIA, 2019). Normalmente, nesse Fórum, o público é composto

Perspectivas em Gestão \& Conhecimento, João Pessoa, v. 10, n. 3, p. 189-204, set./dez. 2020. 
de, pelo menos, um representante de cada Instituição, além de representantes do Ministério da Educação, e pode chegar a, no mínimo, 126 pessoas das IFES, se considerarmos pelo menos um pró-reitor de administração e um pró-reitor de planejamento participante de cada instituição. Entretanto, não é raro ir apenas um representante por instituição, sobretudo em tempos de recursos orçamentários limitados.

A amostra da pesquisa foi composta de 41 pessoas, que aceitaram participar da pesquisa. $O$ instrumento empregado para coletar os dados foi um questionário impresso e simples de preencher, em que foi solicitado o preenchimento dos dados básicos do participante. Em seguida, apresenta-se um conceito de smart campus, além de duas questões do tipo dicotômicas sobre o referido termo e uma questão aberta para os casos em que a resposta anterior fosse positiva. Na sequência, o questionário apresenta 25 afirmações para o participante indicar seu grau de concordância com cada uma delas, em uma escala do tipo Likert (1932) de cinco pontos (Concordo totalmente; Concordo parcialmente; Não concordo nem discordo; Discordo totalmente; Discordo parcialmente). Não foram realizados estudos de correlação. Nessa fase da pesquisa, o tratamento dos dados foi realizado através de estatística descritiva e se limitou a identificar o nível de concordância dos participantes para cada afirmação.

\section{RESULTADOS}

A pesquisa conseguiu alcançar representantes de 27 universidades federais brasileiras, que são apresentadas no Quadro 2:

Quadro 2 - Instituições participantes por estado brasileiro

\begin{tabular}{|c|c|}
\hline Estado & Instituição participante \\
\hline Amapá & Universidade Federal do Amapá (UNIFAP) \\
\hline Bahia & Universidade Federal da Bahia (UFBA) \\
\hline Ceará & Universidade da Integração Internacional da Lusofonia Afro-brasileira (UNILAB) \\
\hline $\begin{array}{l}\text { Distrito } \\
\text { Federal }\end{array}$ & Universidade de Brasília (UnB) \\
\hline $\begin{array}{l}\text { Espírito } \\
\text { Santo }\end{array}$ & Universidade Federal do Espírito Santo (UFES) \\
\hline Goiás & Universidade Federal de Goiás (UFG) \\
\hline $\begin{array}{l}\text { Minas } \\
\text { Gerais }\end{array}$ & $\begin{array}{l}\text { Universidade Federal de Minas Gerais (UFMG) } \\
\text { Universidade Federal de Alfenas (UNIFAL) } \\
\text { Universidade Federal dos Vales do Jequitinhonha e Mucuri (UFVJM) } \\
\text { Universidade Federal de Ouro Preto (UFOP) } \\
\text { Universidade Federal de Itajubá (UNIFEI) } \\
\text { Universidade Federal de São João del-Rei (UFSJ) } \\
\text { Centro Federal de Educação Tecnológica de Minas Gerais (CEFET-MG) } \\
\text { Universidade Federal de Juiz de Fora (UFJF) }\end{array}$ \\
\hline Pará & $\begin{array}{l}\text { Universidade Federal do Pará (UFRPA) } \\
\text { Universidade Federal do Oeste do Pará (UFOPA) }\end{array}$ \\
\hline Paraíba & $\begin{array}{l}\text { Universidade Federal da Paraíba (UFPB) } \\
\text { Universidade Federal de Campina Grande (UFCG) }\end{array}$ \\
\hline Paraná & Universidade Tecnológica Federal do Paraná (UTFPR) \\
\hline
\end{tabular}

Perspectivas em Gestão \& Conhecimento, João Pessoa, v. 10, n. 3, p. 189-204, set./dez. 2020. 


\begin{tabular}{|c|l|}
\hline Pernambuco & Universidade Federal de Pernambuco (UFPE) \\
\hline $\begin{array}{c}\text { Rio de } \\
\text { Janeiro }\end{array}$ & Universidade Federal Rural do Rio de Janeiro (UFRRJ) \\
\hline $\begin{array}{c}\text { Rio Grande } \\
\text { do Norte }\end{array}$ & $\begin{array}{l}\text { Universidade Federal do Rio Grande do Norte (UFRN) } \\
\text { Universidade Federal Rural do Semi-árido (UFERSA) }\end{array}$ \\
\hline $\begin{array}{c}\text { Rio Grande } \\
\text { do Sul }\end{array}$ & $\begin{array}{l}\text { Universidade Federal de Santa Maria (UFSM) } \\
\text { Universidade Federal de Ciências da Saúde de Porto Alegre (UFCSPA) }\end{array}$ \\
\hline $\begin{array}{c}\text { Santa } \\
\text { Catarina }\end{array}$ & Universidade Federal de Santa Catarina (UFSC) \\
\hline Sergipe & Universidade Federal de Sergipe (UFS) \\
\hline
\end{tabular}

Fonte: Dados da pesquisa (2019)

Participaram da pesquisa 41 pessoas - $63 \%$ homens e $37 \%$ mulheres. A maioria (45\%) cursou Especialização; 40\%, Mestrado, 8\%, Doutorado, e 7\% só cursaram a Graduação. As faixas etárias predominantes foram de 36 a 45 anos (25\%) e de 46 a 55 anos (37\%). Esses integrantes são pessoas não são tão jovens, o que poderia indicar um grau de experiência necessário para assumir esses cargos.

As instituições que participaram desta pesquisa apresentaram, pelo menos, um participante. A Tabela 1 apresenta o número de participantes por Instituição.

Tabela 1 - Número de participantes por instituição

\begin{tabular}{|c|c|c|c|}
\hline Instituições & Participantes & Instituições & Participantes \\
\hline UFRRJ & 2 & UFERSA & 1 \\
\hline UFMG & 1 & UFOPA & 1 \\
\hline UFG & 3 & UFCSPA & 1 \\
\hline UNIFAL & 2 & UFOP & 1 \\
\hline UFRN & 3 & UNIFEI & 1 \\
\hline UFCG & 5 & UFSJ & 1 \\
\hline UFRA & 1 & UFES & 1 \\
\hline UFSM & 2 & CEFET-MG & 1 \\
\hline UFVJM & 1 & UFJF & 1 \\
\hline UNILAB & 1 & UFPB & 1 \\
\hline UnB & 2 & UFSC & 2 \\
\hline UFS & 1 & UNIFAP & 1 \\
\hline UFPE & 1 & UTFPR & 2 \\
\hline UFBA & 1 & & \\
\hline
\end{tabular}

A primeira intenção da pesquisa foi de investigar se as pessoas conheciam o conceito de smart campus. Para não haver interpretações equivocadas, inserimos o seguinte conceito de smart campus no questionário: "Smart campus é baseado no uso da tecnologia da

Perspectivas em Gestão \& Conhecimento, João Pessoa, v. 10, n. 3, p. 189-204, set./dez. 2020. 
informação para promover um ambiente de gestão e de aprendizagem mais performante utilizando serviços digitais e sociais." A maioria dos participantes do Fórum $56 \%$ afirmou não conhecer o conceito de smart campus. O Gráfico 1 apresenta a proporção identificada:

Gráfico 1 - Conhecimento sobre smart campus

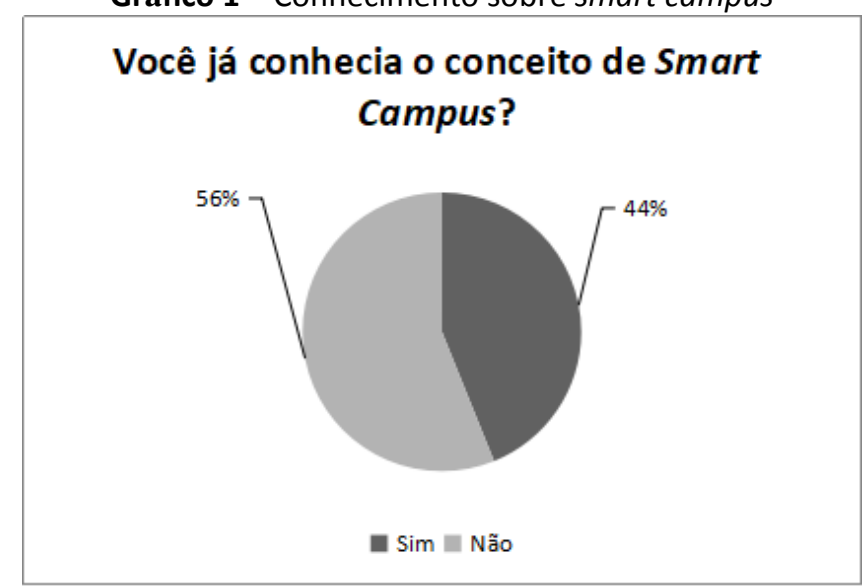

Fonte: Dados da pesquisa (2019)

Sabe-se que o conceito de smart campus é recente e se apresenta como uma ferramenta de alto valor agregado à gestão do campus. Isso porque, devido ao uso de ferramentas inteligentes, o gestor pode reduzir os custos e aumentar a eficiência, como, por exemplo, monitorar e analisar as informações dos estudantes em tempo real, analisando os recursos de imagem que estão sendo armazenados nas nuvens (XIA et al., 2018). Um recurso desse tipo pode reduzir os custos com postos de vigilância e aumentar a segurança ao mesmo tempo. Porém, o fato de a maioria dos gestores de alto escalão das universidades desconhecerem esse conceito é preocupante, sobretudo, nesse momento em que as universidades estão vivendo reduções orçamentárias importantes. As instituições que afirmaram que já estão com o modelo de smart campus implantado ou em andamento foram apenas quatro. $O$ Quadro 3 apresenta as instituições e o ano de criação dos seus respectivos smart campus:

Quadro 3 - Instituições Federais que implantaram o modelo smart campus

\begin{tabular}{|l|l|}
\hline \multicolumn{1}{|c|}{ Instituição } & $\begin{array}{c}\text { Ano de criação do smart } \\
\text { campus }\end{array}$ \\
\hline Universidade Federal de Campina Grande - UFCG & 2019 \\
\hline Universidade Federal do Rio Grande do Norte - UFRN & 2016 \\
\hline Universidade Federal do Espírito Santo - UFES & 2016 \\
\hline Universidade Federal de Santa Maria - UFSM & 2016 \\
\hline
\end{tabular}

Fonte: Dados da pesquisa (2019)

Participaram da pesquisa 41 representantes de 27 Universidades, todavia apenas quatro delas afirmaram ter implantado o modelo de smart campus: a UFCG, a UFRN, a UFES e a UFSM. Algumas mais adiantadas do que outras, porém todas em processo de implantação ou adaptação de modelagem. Os dirigentes afirmaram que os smart campus são uma criação recente. Segundo eles, o modelo tem como foco abranger a gestão financeira, a avaliação institucional, a gestão do ensino de graduação, a eficiência energética, o gerenciamento da frota e o gerenciamento e o monitoramento da segurança do campus com o uso de 
tecnologias oriundas da Internet das Coisas. Apesar de serem importantes, os smart campus ainda não são muito representativos em um universo de 63 universidades públicas federais brasileiras.

Quadro 4 - Dimensões observadas no questionário

\begin{tabular}{|c|c|}
\hline $\begin{array}{l}\text { Dimensões } \\
\text { observadas }\end{array}$ & Frases/percentual de concordância \\
\hline Importância & $\begin{array}{l}\text { - É importante criar um smart campus ( } 98 \% \text { concordam totalmente). } \\
\text { - A criação de um smart campus é uma forma de melhorar a gestão da } \\
\text { - Enstituição ( } 90 \% \text { concordam totalmente). } \\
\text { ( } 71 \% \text { vejo o smart campus como algo necessário para a minha Instituição } \\
\text { - Eu acredito que criar um smart campus vai melhorar a gestão do } \\
\text { campus, o ensino e a aprendizagem ( } 68 \% \text { concordam totalmente). } \\
\text { - A sociedade vê de forma positiva um smart campus ( } 37 \% \text { concordam } \\
\text { totalmente, e } 29 \%, \text { parcialmente). }\end{array}$ \\
\hline $\begin{array}{c}\text { Conhecimento dos } \\
\text { gestores sobre smart } \\
\text { campus }\end{array}$ & $\begin{array}{l}\text { - Eu sei como criar um smart campus na minha Instituição ( } 20 \% \\
\text { discordam totalmente, e } 27 \% \text {, parcialmente) } \\
\text { - Atualmente estou envolvido no projeto de criação do smart campus na } \\
\text { minha Instituição ( } 41 \% \text { discordam totalmente, e } 32 \% \text {, parcialmente). } \\
\text { - Eu já tive experiência com a criação de um smart campus (56\% } \\
\text { discordam totalmente, e } 24 \% \text {, parcialmente) } \\
\text { - Estou pronto para fazer o que for necessário para criar um smart } \\
\text { campus na minha Instituição ( } 34 \% \text { concordam totalmente, e } 34 \% \text {, } \\
\text { parcialmente). }\end{array}$ \\
\hline $\begin{array}{l}\text { Estrutura da } \\
\text { instituição }\end{array}$ & $\begin{array}{l}\text { - A minha Instituição tem estrutura para criar um smart campus ( } 34 \% \\
\text { - } \quad \text { As tecnologias desenvolvidas na Instituição devem servir também para } \\
\text { atender às demandas internas próprias do órgão, sobretudo, no } \\
\text { tocante a sua gestão ( } 95 \% \text { concordam totalmente). } \\
\text { - Criar um smart campus na minha Instituição é um grande desafio (56\% } \\
\text { concordam totalmente) } \\
\text { - Na minha Instituição, existem ações isoladas que se enquadram no } \\
\text { smart campus ( } 39 \% \text { concordam totalmente, e } 39 \%, \text { parcialmente). }\end{array}$ \\
\hline Gestão & $\begin{array}{l}\text { - O smart campus reduz os custos da gestão ( } 59 \% \text { concordam } \\
\text { totalmente). } \\
\text { O smart campus é uma estratégia para a crise orçamentária das } \\
\text { Instituições públicas de ensino superior ( } 44 \% \text { concordam totalmente, e } \\
46 \%, \text { parcialmente). } \\
\text { - Eu vejo a criação de um smart campus como um modelo que deverá ser } \\
\text { adotado por todas as Instituições públicas de ensino superior ( } 68 \% \\
\text { concordam totalmente). } \\
\text { Ações conjuntas entre as Instituições de ensino são necessárias para } \\
\text { criar um modelo de smart campus adaptado para a realidade brasileira } \\
\text { ( } 71 \% \text { concordam totalmente). } \\
\text { As boas práticas existentes já devem ser compartilhas através de uma } \\
\text { ferramenta que favoreça a criação do conhecimento ( } 88 \% \text { concordam } \\
\text { totalmente). }\end{array}$ \\
\hline Equipe & $\begin{array}{l}\text { - Os servidores da minha Instituição têm abertura para usar novas } \\
\text { tecnologias ( } 24 \% \text { concordam totalmente, e } 68 \% \text {, parcialmente). } \\
\text { - A minha equipe tem condições de criar um smart campus ( } 20 \% \\
\text { concordam totalmente, e } 39 \%, \text { parcialmente). } \\
\text { - A minha equipe é aberta a novos desafios ( } 34 \% \text { concordam totalmente, }\end{array}$ \\
\hline
\end{tabular}

Perspectivas em Gestão \& Conhecimento, João Pessoa, v. 10, n. 3, p. 189-204, set./dez. 2020. 


\begin{tabular}{|c|c|}
\hline & $\begin{array}{l}\text { e } 54 \%, \text { parcialmente). } \\
\text { - Colocarei todo o meu esforço para criar um smart campus na minha } \\
\text { Instituição ( } 32 \% \text { concordam totalmente, e } 39 \% \text {, parcialmente). }\end{array}$ \\
\hline $\begin{array}{l}\text { Resultados } \\
\text { esperados do smart } \\
\text { campus }\end{array}$ & $\begin{array}{l}\text { - Com a criação do smart campus, a Instituição poderá promover, de } \\
\text { forma concreta, a inovação e o empreendedorismo em todas as } \\
\text { camadas da comunidade acadêmica ( } 54 \% \text { concordam totalmente). } \\
\text { - O smart campus é uma forma de criar ações concretas de } \\
\text { sustentabilidade ( } 68 \% \text { concordam totalmente). } \\
\text { - Eu acredito que criar um smart campus proporcionará o } \\
\text { desenvolvimento de novas parcerias e novas fontes de recursos para a } \\
\text { Instituição ( } 54 \% \text { concordam totalmente). }\end{array}$ \\
\hline
\end{tabular}

Fonte: Dados da pesquisa (2019)

A análise dos resultados está organizada na mesma ordem da apresentação dos resultados:

- Importância; Os gestores veem o modelo de smart campus como importante e necessário para as universidades federais brasileiras. Entretanto, preocupam-se sobre como fazê-lo. A literatura também enfatiza a importância do smart campus, bem como, a emergência do tema (WANG et al., 2018; XIA et al., 2018). Trata-se um modelo novo, onde as tecnologias estão sendo testadas em diversas áreas do campus (TORRES-SOSPEDRA et al., 2015; ROMANO et al., 2016; CHEN \& ZHANG, 2012; DE ANGELIS et al., 2015; DE SÁ et al., 2016; BANDARA et al., 2016). A combinação de tecnologias ainda não é evidente na literatura para uso gerencial. Percebe-se uma explicação detalhada de algoritmos e tecnologias enfatizando as vantagens e aplicações de cada uma delas de maneira isolada.

- Conhecimento dos gestores sobre smart campus; A maioria dos gestores apontou a falta de conhecimento sobre como criar um smart campus. A gestão necessita de um modelo que integre as necessidades dos gestores públicos. Nesse caso, o fator novo desse modelo atrai mas ao mesmo tempo provoca hesitação dos gestores sobre a sua aplicabilidade. Os desafios próprios das organizações burocráticas não facilitam as tomadas de decisão. Entretanto os problemas enfrentados pela gestão tradicional podem ser solucionados ou amenizados pela criação de um smart campus (RIBEIRO, 2014; YANG et al., 2018). Entretanto, os estudos realizados apresentam dimensões importantes que podem ser consideradas pelos gestores para a criação de um smart campus adaptado às necessidades do campus considerando as suas próprias características locais ou regionais.

- Estrutura da instituição; Quanto à estrutura, eles disseram que têm a estrutura necessária para criar um smart campus, mas admitem que não têm conhecimento sobre como criá-lo e entenderem que é um grande desafio para as IFEs. Para se criar um smart campus é necessário um investimento em infraestrutura (ATIF et al., 2015; BARBATO et al., 2016; XIN et al., 2016; BATES; FRIDAY, 2017). Certamente isso irá variar de uma Instituição para outra, cabe-se fazer um levantamento dos recursos existentes e necessários para propor um plano de implantação desse modelo com perspectivas e resultados esperados.

- Gestão; Eles reconhecem que o modelo de smart campus é uma solução estratégica para combater os problemas atuais enfrentados pelas IFEs, no tocante a sua gestão, entendem que essas instituições apresentam desafios e estruturas muito parecidas e veem com bons olhos um modelo que englobe e compartilhe os desafios e as oportunidades dessas instituições. Isso pode ser explicado pela abertura mais colaborativa que as Instituições estão adotando. A crescente demanda dos órgãos e 
controle para reduzir custos ou justificar os gastos demandam diferentes pareceres técnicos que estão sendo cada vez mais sendo compartilhados com os servidores no Brasil, através de grupos formados com esse objetivo. A governança também é um tema bastante explorado no serviço público e valoriza o uso de ferramentas que a deixem mais transparente e ágil (MELLO; SILVA, 2018; INSTITUTO BRASILEIRO DE GOVERNANÇA CORPORATIVA, 2018).

- Equipe; os gestores também afirmaram que confiam no potencial de suas equipes e acreditam que elas são capazes de atuar em um ambiente colaborativo, como o smart campus, e que se empenhariam para criar um smart campus. Os gestores afirmam que a equipe tem condições ou competências para enfrentar um projeto dessa amplitude. Essa é uma dimensão importante para a implantação desse tipo de modelo. Trata-se de uma proposta inovadora e com muitas aplicações de internet das coisas e inteligência artificial, consequentemente, a geração de big data. Então, pessoas capacitadas para lidar com essas tecnologias favorece as previsões e as tomada de decisão dos gestores.

- Resultados esperados do smart campus; Os gestores acreditam que podem alcançar resultados qualitativos e quantitativos com a criação do smart campus, que englobam fatores como, inovação, sustentabilidade e aumento de recursos orçamentários e financeiros (AI-MIN et al., 2018; XIN et al., 2018; ZHIHUA et al., 2018; LUO, 2018; PRANDI et al., 2019).

O conceito de smart campus está intrinsecamente embasado nas tecnologias da Informação e da comunicação e se harmoniza com as abordagens de foco tecnológico. No tocante, a gestão não identificamos nos estudos sobre smart campus nenhum que englobasse modelos próprios das ciências administrativas. Todas as propostas de soluções tecnológicas atendem a uma parte ou um segmento das atividades do campus ou necessidades relacionadas ao estilo de vida dos estudantes. Percebeu-se que existe uma preocupação em gerenciar o alto volume de dados produzidos nessas estruturas, assim como na armazenagem e na segurança da informação. Entretanto, ainda se faz necessário uma proposta de modelo de gestão na perspectiva de inteligência tecnológica de maneira ampla para os campi de universidades federais. Os gestores necessitam de algo claro e pragmático para que eles possam implantar determinado modelo no serviço público. Existem vários desafios administrativos e legais que exigem muita responsabilidade nas tomadas de decisão. Por isso, é importante um modelo claro e transparente que ajude a gestão, favoreça a colaboração e a transparência bem como a relação com a sociedade.

A percepção dos gestores sobre o conceito de smart campus e sua criação é positiva. 0 smart campus é um conceito emergente e em construção, que ainda apresenta vários questionamentos sobre sua aplicabilidade do ponto de vista das tecnologias e da gestão. A gestão pública muitas vezes apresenta uma alta rotatividade dos gestores, isso resulta em trabalhos incompletos que tendem a ser descartados pela gestão seguinte. Um projeto de gestão baseado em tecnologias inteligentes como o smart campus deve ser um projeto institucional com uma estrutura administrativa própria, ou seja, poderia ser incorporado na Pró-Reitoria de Gestão, Pró-Reitoria de Planejamento ou na Pró-Reitoria de Infraestrutura.

Os resultados da pesquisa são bastante encorajadores, pois revelaram que o grupo formado por 41 gestores de 27 instituições brasileiras de ensino superior demonstraram entender a importância da criação do smart campus no atual cenário econômico. Trata-se de um grupo bastante representativo no cenário nacional, que agrega um alto valor aos resultados da pesquisa, pois trouxe materialidade para debater sobre um modelo que pode ser uma alternativa para a atual gestão das instituições de ensino superior de uma perspectiva

Perspectivas em Gestão \& Conhecimento, João Pessoa, v. 10, n. 3, p. 189-204, set./dez. 2020. 
sistemática e global. Esses pró-reitores tomam decisões estratégicas, representam o alto escalão das instituições de ensino e são experts em Administração e Contabilidade pública. Eles são responsáveis, entre outras atribuições, pela execução orçamentária dessas instituições.

Por isso, a percepção deles sobre a implantação de um novo modelo de gestão é fundamental, porque são eles que viabilizam a cooperação interorganizacional, a aquisição de bens e serviços e a implantação de procedimentos legais para atingir os objetivos organizacionais em todos os níveis da instituição. Seguindo a perspectiva proposta por Mello e Silva (2018), os pró-reitores é que deveriam implantar a estrutura de governança adequada para o campus inteligente, englobando os stakeholders.

\section{CONSIDERAÇÕES FINAIS}

O conceito de smart campus ainda carece de abordagens gerenciais. Trata-se de uma proposta que tem um alto valor agregado para a comunidade universitária em geral. As abordagens ainda estão concentradas nas áreas das ciências duras, por isso é necessário um modelo de gestão inteligente, que englobe essa realidade tecnológica para beneficiar o ensino, a comunicação, a inovação, a segurança etc. Os Campi das IFEs apresentam uma estrutura gerencial complexa, burocrática e com alta rotatividade dos gestores do nível estratégico. Esses desafios freiam a formação e o desenvolvimento de competências próprias para esse cenário organizacional.

As fraquezas gerenciais devem ser elencadas e consideradas na plataforma smart campus, com o objetivo de promover a transparência, a gestão da informação e a participação de diferentes grupos de interesses nas tomadas de decisões de maneira ágil, plena e equitável. Portanto um modelo de governança sistemático, acessível e adaptado é necessário para contemplar a abordagem inteligente proposta no modelo de smart campus. As parcerias e os financiamentos que viabilizem o desenvolvimento de tecnologias que englobem cada perfil de usuário, estudante, professor e técnicos administrativos.

Considerando que a pesquisa apresentou limitações metodológicas, uma abordagem qualitativa seria apreciada, por meio de uma entrevista semiestruturada com os gestores, e teria possibilitado entender os pontos fortes e os fracos de cada instituição, proporcionando uma pista de pesquisa maior para o desenvolvimento de um modelo de gestão baseado nas especificidades de cada uma delas. As próximas pesquisas poderiam detalhar a estrutura administrativas das Instituições e apresentar uma proposta de gestão compartilhada para os smart campus das instituições públicas de ensino superior no Brasil.

\section{REFERÊNCIAS}

AI-MIN, Y.; LI, S.; REN, C. HUAN, L.; HUI-XIANG; H.; YANG; L. Situational Awareness System in the Smart Campus. IEEE Access, 2018.

ATIF, Y.; MATHEW, S.S.; LAKAS, A. Building a smart campus to support ubiquitous learning. Journal of Ambient Intelligence and Humanized Computing, n. 6, p. 223-238, 2015. DOI 10.1007/s12652-014-0226-y.

BANDARA, H. M. A. P. K.; JAYALATH, J. D. C.; BANDARANAYAKE, A. U.; MARAIKAR, Z.; RAGEL, R. G. Smart campus phase one: Smart parking sensor network, Manufacturing \& Industrial Engineering Symposium (MIES), Colombo - Sri Lanka, n. 1, v. 6. 2016. http://dx.doi.org/10.1109/MIES.2016.7780262

Perspectivas em Gestão \& Conhecimento, João Pessoa, v. 10, n. 3, p. 189-204, set./dez. 2020. 
BARBATO, A. Energy optimization and management of demand response interactions in a smart campus. Energies, v. 9, n. 6, p. 398, 2016.

BATES, O.; FRIDAY, A. Beyond data in the smart city: repurposing existing campus IoT. IEEE Pervasive Computing, v. 16, n. 2, p. 54-60. 2017.

BOCCATO, V. R. C. Metodologia da pesquisa bibliográfica na área odontológica e o artigo científico como forma de comunicação. Revista de Odontologia da Universidade de São Paulo, v. 18, n. 3, p. 265-274, 2006.

CHEN, Y.; ZHANG, R.; ZHANG, S. Service encapsulation-based model for Smart Campus. Journal of Electronic Commerce in Organizations, 2012.

DE ANGELIS E.; CIRIBINI, A. L. C.; TAGLIABUE, L. C.; PANERONI, M. The Brescia Smart Campus Demonstrator. Renovation toward a zero Energy Classroom Building. Procedia Engineering, n.118, p. 735-743, 2015. https://doi.org/10.1016/j.proeng.2015.08.508

DE SÁ, C. M.; PARADA, L.; STRENGARI, L. A.; BERTONI L. A.; HELLMEISTER, M. S.; MARINS, K. R. C. C. Smart Campus: proposta de aplicação de conceitos inteligentes na cidade universitária "Armando de Salles Oliveira". In: XVI Encontro Nacional de Tecnologia do Ambiente Construído. Anais...Desafios e perspectivas da internacionalização da construção. São Paulo, 2016.

FACENS. Disponível em: https://smartcampus.facens.br/. Acesso em 06 Jun. 2019.

FONSECA, J. J. S. Metodologia da pesquisa científica. Fortaleza: UEC, Apostila, 2012.

GIBSON, D. V.; KOZMETSKY, G.; SMILOR, R. W. The Technopolis Phenomenon: Smart Cities, Fast Systems, Global Networks. Rowman \& Littlefield, New York (SMART CITY COUNCIL), 1992. Disponível em: https://smartcitiescouncil.com/.

GIL, A. C. Como elaborar projetos de pesquisa. São Paulo: Atlas, 2008. Disponível em: https://aspuv.org.br/cortes-orcamentarios-ameacam-instituicoes-federais-de-ensino/ https://educacao.estadao.com.br/noticias/geral,com-fim-do-ciencia-sem-fronteirasintercambio-em-graduacao-cai-ate-99,70002090320. Acesso em: 06 Jun. 2019.

INSTITUTO BRASILEIRO DE GOVERNANÇA CORPORATIVA. Relatório Anual Ecossistema de Governança: Inovação e legado. 2018.

JIWEI, Z. Spatio-Temporal Association Query Algorithm for Massive Video Surveillance Data in Smart Campus. IEEE Access, 2018.

KWOK, Lam-for. A vision for the development of i-campus lam for work. Smart Learning environments, 2015.

LIKERT, R. A Technique for the Measurement of Attitudes. Archives of Psychology, v. 140, p. 1$55,1932$.

Perspectivas em Gestão \& Conhecimento, João Pessoa, v. 10, n. 3, p. 189-204, set./dez. 2020. 
LUO, L. Data Acquisition and Analysis of Smart Campus Based on Wireless Sensor. Wireless Personal Communications.

MASSACHUSETTS INSTITUTE OF TECHNOLOGY, 2018. Disponível em: http://www.mit.edu/.

MELLO, M. S.; SILVA, R. R. O. Práticas de governança corporativa em instituições de ensino superior: um estudo de caso em uma universidade de Santa Catarina. Revista de

Administração e Contabilidade - RAC (CNEC). Ano 17, n. 33, p.68-80, jan./jun., 2018.

NAN, Feng. Real-Time Monitoring of Smart Campus and Construction of Weibo Public Opinion Platform. IEEE Access, 2018.

PORTAL DA TRANSPARÊNCIA. Disponível em: http://www.portaltransparencia.gov.br/. Acesso em 06 Jun. 2019.

PRANDI, C.; MONTI, L.; CECCARINI, C.; SALOMONI, P. Smart campus: fostering the community awareness through an intelligent environment. Mobile networks and applications, 2019.

RIBEIRO, R. M. C. Os desafios contemporâneos da gestão universitária: discursos politicamente construídos, 2014. Disponível em:

http://www.anpae.org.br/ibero americano iv/eixo2.html. Acesso em 06 Jun. 2019.

ROMANO, R. R.; SIRON, L. G. P. P.; PACHECO, C. P. Smart Campus FACENS - Construindo uma cidade inteligente em um Campus Universitário utilizando-se do FabLab, 2016. Disponível em: http://fablearn.org/wp-content/uploads/2016/09/FLBrazil2016paper150.pdf. Acesso em 06 Jun. 2019.

SÁNCHEZ-TORRES, B. et al. Smart Campus: trends in cybersecurity and future development. Revista Facultad de Ingeniería, 2018.

SILVA, E. L.; MENEZES, E. M. Metodologia da pesquisa e elaboração de dissertação. 4. ed. Florianópolis: UFSC, 2005. 138 p. Disponível em:

<www.posarq.ufsc.br/download/metPesq.pdf>. Acesso em: 02 jun. 2020.

SMART CITY COUNCIL. Disponível em: https://smartcitiescouncil.com/. Acesso em: 06 Jun. 2019.

TORRES-SOSPEDRA J. Enhancing integrated indoor/outdoor mobility in a smart campus.

International Journal of Geographical Information Science, v. 29, n. 11, p. 1955-1968, 2015.

Disponível em: https://www.tandfonline.com/doi/full/10.1080/13658816.2015.1049541.

WANG, L. Research on a Dynamic Virus Propagation Model to Improve Smart Campus Security. IEEE Access, 2018.

XIA, Z. et al. Secure Image LBP Feature Extraction in Cloud-Based Smart Campus. IEEE Access, 2018.

Perspectivas em Gestão \& Conhecimento, João Pessoa, v. 10, n. 3, p. 189-204, set./dez. 2020. 
XIN, D.; KONG, X.; ZHANG, F.; CHEN, Z.; KANG, J. On Campus: a mobile platform towards a smart campus. SpringerPlus, 2016.

XIN, X.; WANG, Y.; YU, S. Teaching Performance Evaluation in Smart Campus. IEEE Access, 2018.

YANG, Ai-Min. Situational Awareness System in the Smart Campus. IEEE Access. 2018.

YI L.; ZIXI C. Intelligent and Real-Time Data Acquisition for Medical Monitoring in Smart Campus. IEEE Access, v. 6, p. 74836-74846, 2018. doi: 10.1109/ACCESS.2018.2883106.

YING, C.; ZHANG, R.; ZHANG, S.; CHEN, Y. Service encapsulation-based model for Smart Campus. Journal of Electronic Commerce in Organizations, 2012.

YU, W.; ZHANG, N.; XIA, C. Multi-Agent-Based Unsupervised Detection of Energy Consumption Anomalies on Smart Campus. IEEE Access, 2019.

ZHIHUA, X.; MA, X.; SHEN, Z.; SUN, X.; XIONG, N. N; JEON, B. Secure Image LBP Feature Extraction in Cloud-Based Smart Campus. IEEE Access, 2018.

ZHIHONG, T.; CUI, Y.; AN, L.; SU, S.; YIN, X.; YIN, L.; CUI, X. A Real-Time Correlation of HostLevel Events in Cyber Range Service for Smart Campus. IEEE Access, 2018.

Artigo recebido em 27/10/2019 e aceito para publicação em 11/09/2020

Perspectivas em Gestão \& Conhecimento, João Pessoa, v. 10, n. 3, p. 189-204, set./dez. 2020. 